\title{
Mama tuberosa: clasificación y alternativas quirúrgicas en los últimos años
}

\section{Tuberous breast: classification and surgical alternatives in the last years}

Celeste N. Di Pietro

REVISTA ARGENTINA DE CIRUGÍA PLÁSTICA 2018;24(2):88-94. DOI/10.32825/RACP/201802/0088-0094

\section{ÍNDICE}

1. Resumen/Summary.. . .3

2. Introducción................................................................4

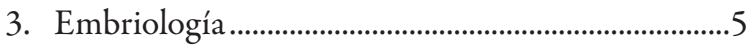

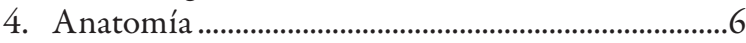

5. Presentación clínica .......................................................7

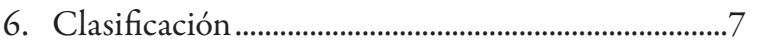

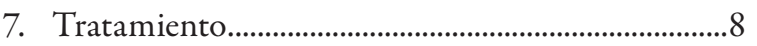

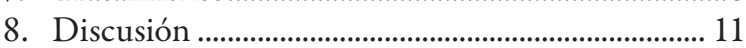

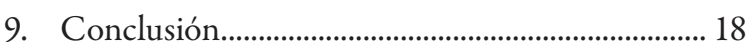

10. Bibliografía ............................................................... 19

\section{RESUMEN}

La mama tuberosa constituye una anomalía congénita de la mama que afecta a mujeres jóvenes unilateral o bilateralmente, manifestándose en la pubertad con el crecimiento de la mama y presentando un impacto psicológico significativo en las pacientes. Se caracteriza por una deficiencia en las dimensiones horizontal y vertical de la mama, constricción de la base mamaria, hipoplasia parenquimatosa, deficiencia de piel del polo inferior, malposición superior del surco submamario, hernia areolar y asimetría. Debido a la gran variedad de formas de presentación, la mama tuberosa ha sido tratada quirúrgicamente con numerosos tipos de técnicas por diferentes cirujanos a lo largo del tiempo. No se ha llegado a un consenso sobre la mejor técnica quirúrgica. Con una correcta clasificación y evaluación preoperatoria adecuada se puede llegar a un planeamiento quirúrgico más acertado.

\footnotetext{
1. Rotación Cirugía Estética. Universidad del Salvador

$\triangle$ Correspondencia: Dra. Celeste N. Di Pietro. celestedipietro@hotmail.com
}

Los autores no declaran conflictos de intereses

Recibido: 09/09/2018 / Aceptado: 10/10/18
Palabras clave: mama tuberosa, anomalías congénitas de la mama, clasificación, tratamiento quirúrgico de mama tuberosa.

\section{SUMMARY}

Tuberous breast is a congenital anomaly of the breast affecting young women unilaterally or bilaterally, manifesting in puberty with the growth of the breast and presenting a significant psychological impact on patients. It is characterized by a deficiency in the horizontal and vertical dimensions of the breast, contricted base,underdevelopment of the breast, skin deficiency of the lower pole, superiorly malpositioned submammary fold, areolar herniation and asymmetry. Due to the great variety of presentation forms, the tuberous breast has been treated surgically with many types of techniques by different surgeons over time. No consensus has been reached on the best surgical technique. With a correct classification, and adequate preoperative evaluation, it is possible to arrive at a more successful surgical planning.

Keywords: tuberous breast, congenital anomalies of breast, classification, surgical treatment of tuberose breast.

\section{INTRODUCCIÓN}

Bajo la denominación de mama tuberosa conocemos una anomalía de la glándula mamaria femenina, unilateral o bilateral, descripta por primera vez por Rees y Aston en $1976^{1}$. Se utilizan varios nombres para describir a esta anomalía congénita de la mama, por ejemplo: mama tuberosa; mama tubular; mama de Snoopy; hernia del complejo areolar; mama de base estrecha; hipoplasia de polo inferior ${ }^{2}$, son en realidad la misma entidad anatómica y clínica: una anomalía mamaria con diversos grados de severidad o desarrollo ${ }^{3}$.

La mama tuberosa se manifiesta durante la pubertad y representa para el paciente un problema de gran frustración; por lo tanto, no corrigiendo esta deformidad 


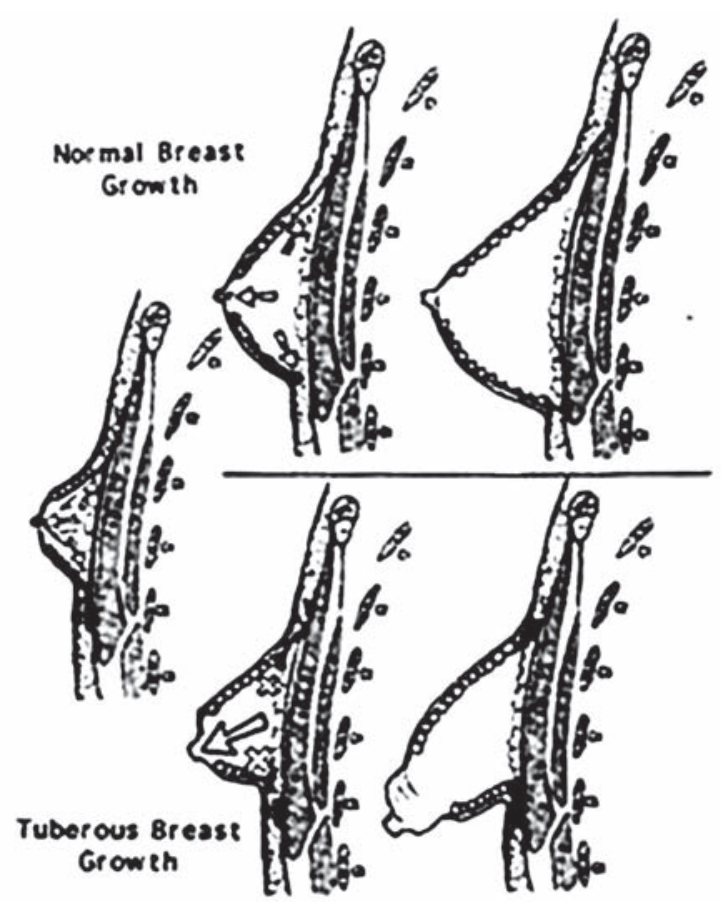

Figura 1. Arriba: desarrollo normal de la mama. Abajo: desarrollo de mama tuberosa (3).

en el tiempo puede llevar a grandes consecuencias en la vida de las pacientes, como baja autoestima, sensación de inseguridad, e incluso una personalidad perturbada ${ }^{4}$.

No se conoce ninguna etiología para este síndrome ${ }^{5}$. La esencia de la deformidad es la alteración de las dimensiones verticales y horizontales de la mama en general ${ }^{6}$. Las características incluyen constricción de la base mamaria, hipoplasia parenquimatosa, deficiencia de piel del polo inferior, malposición superior del surco submamario, hernia areolar y asimetría ${ }^{7}$.

La corrección de esta anomalía, en sus diferentes grados, es exclusivamente quirúrgica. No existe una técnica quirúrgica única adecuada para corregir todos los tipos de las diferentes deformidades; sin embargo, podemos trazar un denominador común, como: transferir el tejido dislocado, conservado, o constreñido a través de colgajos glandulares, que corregirán la deformidad existente y al mismo tiempo permiten abrir y ampliar la base mamaria conservada y fibrótica constreñida ${ }^{8}$.

\section{EMBRIOLOGÍA}

Desde el punto de vista del desarrollo embriológico el brote mamario se diferencia y forma durante la vida intrauterina hasta el nacimiento ${ }^{2}$ pero su desarrollo pleno como órgano mamario se produce al momento de la pubertad. Este período puede variar de 7-9 a 15-16 años de edad dependiendo de las diferentes características raciales y constitucionales?

Según la mayoría de las opiniones, el brote mamario embrionario está restringido en su desarrollo a un área pequeña de la pared torácica por un anillo fibro-

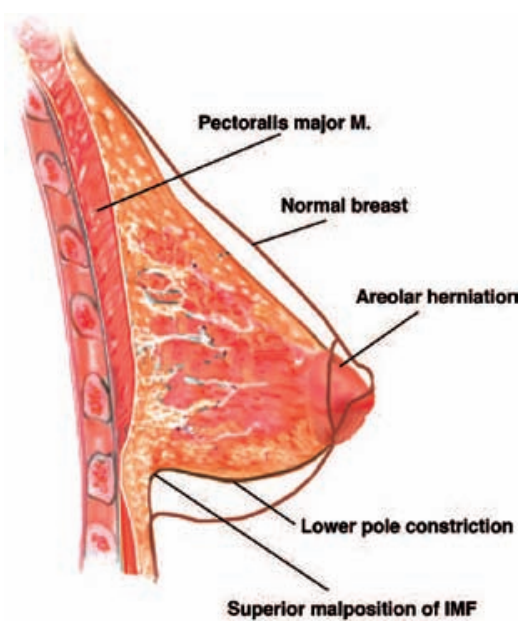

Figura 2. Características anatómicas de mama tuberosa (7).

so constrictivo. Este anillo constrictivo impide la expansión periférica del parénquima mamario que, como consecuencia, se expande en la única dirección disponible, es decir, hacia adelante, a través de la areola que es más elástico que la piel circundante y, por lo tanto, un sitio de expansión. Las bandas fibrosas rodean toda la base periférica de la mama, contribuyendo a la mala posición superior y estrechez del pliegue inframamario. El anillo fibroso constrictivo es una consecuencia de un engrosamiento de la fascia superficial. La fascia superficial está ausente en la región areolar por lo que todo el parénquima mamario está herniado a través de esta región muy elástica ${ }^{2,3}$ (Figura 1).

\section{ANATOMÍA}

Como resultado del origen ectodérmico de la mama y su invaginación en el mesénquima subyacente, el tejido mamario está contenida dentro de una cubierta fascial (la fascia superficial) que es continua con la fascia abdominal o fascia de Camper que, a su vez, tiene dos capas: la superficial que cubre el parénquima mamario y la profunda que forma el límite posterior del parénquima mamario y en contacto con la fascia profunda de los músculos pectoral mayor y serrato anterior. Entre la capa superficial y la profunda de la fascia existen unos tractos fibrosos (ligamentos suspensorios de Cooper) que unen ambas capas y que se extienden hasta la dermis de la piel que recubre la mama y a la fascia pectoral profun$\mathrm{da}^{10,11,12}$. Un hecho a destacar en las mamas tuberosas es la ausencia de la capa superficial de esta fascia en la zona situada bajo la areola, junto con un engrosamiento de la fascia superficial especialmente en el polo inferior de la mama ${ }^{13}$. En las mamas tuberosas existe una banda de constricción, situada periféricamente al complejo areola-pezón, que altera el normal desarrollo de la mama. El resultado final de todo ello es que la mama no puede desarrollarse hacia el polo inferior $\mathrm{y}$, al tener un punto de menor resistencia a nivel de la areola por la falta de esta 

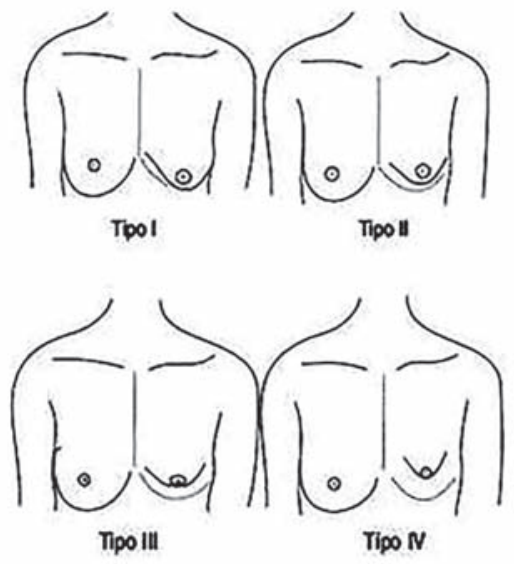

Figura 3. Clasificación Von Heimburg

capa fascial, la glándula en crecimiento se hernia hacia el complejo areola-pezón ${ }^{2}$ (Figura 2).

\section{PRESENTACIÓN CLÍNICA}

Las diferentes anomalías que se pueden encontrar las podemos concretar en ${ }^{5}$ :

Alteración de la base de implantación de la mama y constricción del surco submamario, que suele estar más elevado y ser más corto.

Alteración en el crecimiento mamario,que se efectúa de manera preferente en los cuadrantes superiores, con falta de desarrollo de los inferiores, lo que le da el aspecto tubular a la mama.

Alteración en el tamaño de la placa areolar, que cede bajo la presión de la constricción, dando lugar a una hernia areolar de la glándula. Esta Hernia no siempre está presente, es más frecuente en grados severos.

Anomalías del volumen mamario, siendo la mayoría hipotróficas, salvo en el grado I.

Asimetría mamaria, que está presente en más de $2 / 3$ de los casos.

Anomalía bilateral: las dos mamas son generalmente tuberosas y todos los grados pueden estar asociados.

\section{CLASIFICACIÓN}

\section{CLASIFICACIÓN VON HEIMBURG(14) (Figura 3):}

- TIPO I: hipoplasia del cuadrante medial inferior.

- TIPO II: hipoplasia del cuadrante medial inferior y lateral, con suficiente piel en región subareolar.

- TIPO III: hipoplasia del cuadrante medial inferior y lateral, con insuficiente piel en región subareolar.

- TIPO IV: hipoplasia de los cuatro cuadrantes mamarios. Constricción mamaria severa tanto en eje vertical como horizontal, con base mamaria mínima.

\section{CLASIFICACIÓN DE GROLLEAU Y COLS. (3 (Figu-} ra 4): modificó la clasificación de Von Heimburg para describir solo 3 tipos ya que no se pudo ver diferencia objetiva o clínica entre los tipos II y III.
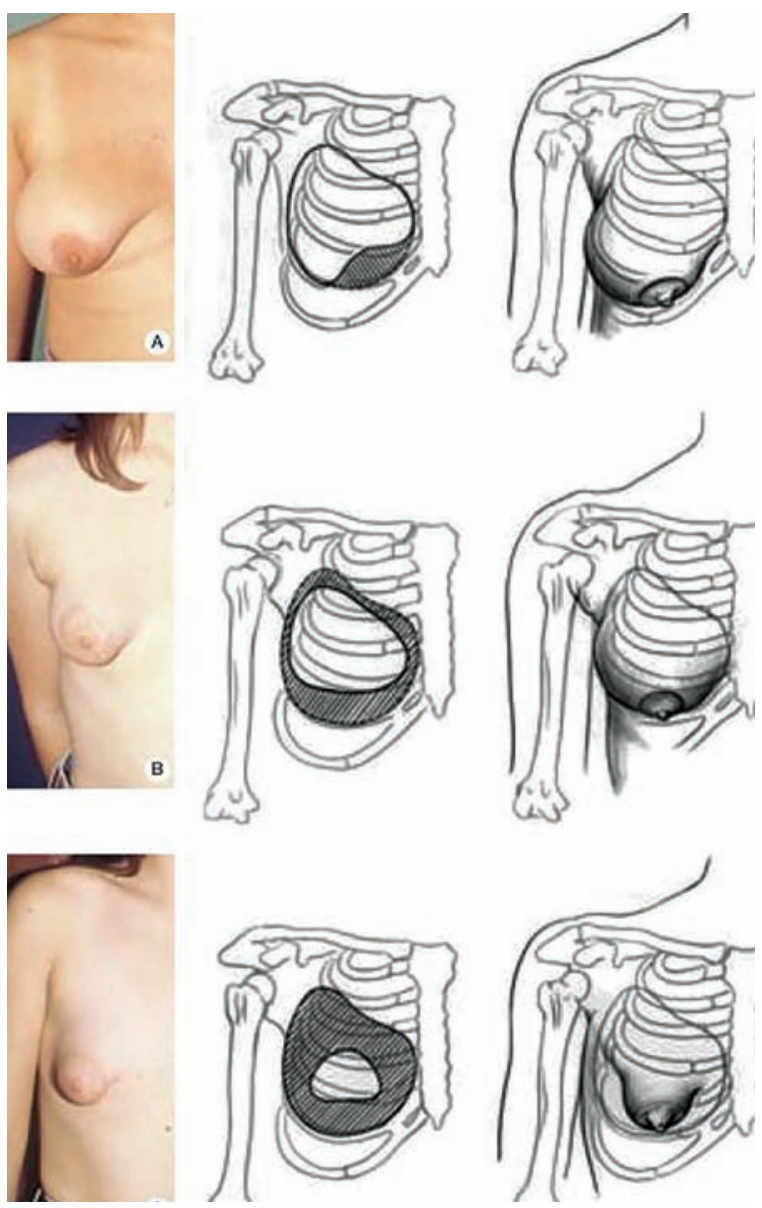

Figura 4. Clasificación Grolleau y cols.

- TIPO I: la falta de desarrollo se limita al cuadrante infero-interno. La areola se encuentra desviada hacia abajo y adentro, siendo el volumen de la mama normal o hipertrófica.

- TIPO II: los dos cuadrantes inferiores son deficientes en su desarrollo. En estos casos la areola está desviada mirando hacia abajo.

- TIPO III: todos los cuadrantes están afectados y son deficientes, la base mamaria se encuentra retraída y la mama tiene un aspecto de tubérculo o caprino.

\section{TRATAMIENTO}

El tratamiento de la deformidad mamaria tuberosa generalmente debe enfocarse en tres áreas principales. En primer lugar, la envoltura de la piel restringida con el surco submamario mal posicionado, debe normalizarse tanto como sea posible. Segundo, las relaciones anatómicas que resultan en la hernia areolar deben ser corregidas. Y, finalmente, debe abordarse cualquier asimetría en el tamaño o la forma de los senos. En la mayoría de los casos, es necesario combinar estas estrategias quirúrgicas para optimizar el resultado final. El manejo quirúrgico de la mama tuberosa se determina en última instancia por la gravedad de la presentación, y requiere que el cirujano y el paciente aborden cada 

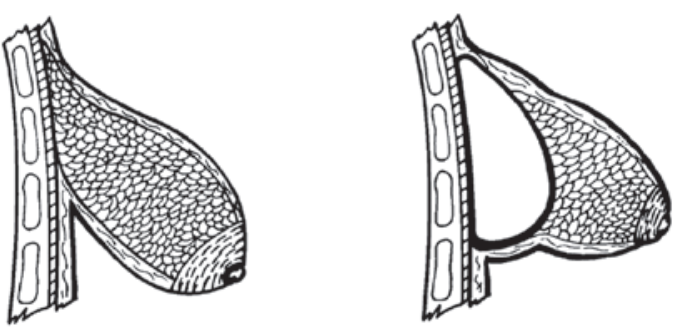

Figura 5.

aspecto de la anomalía. Los principios del tratamiento deben incluir lo siguiente ${ }^{15}$ :

1. Liberación de la base contraída tanto en un plano vertical como horizontal.

2. Restaurar un pezón normal a la distancia del surco submamario mediante una combinación de mastopexia y reducción del surco submamario.

3. Eliminar el antiguo surco submamario para evitar una apariencia de doble burbuja.

4. Reducción del tejido herniado.

5. Corrección de la hipertrofia de areola.

6. Restauración del volumen mamario.

7. Corrección de la asimetría mamaria.

\section{BASE CONSTRICTA Y SURCO SUBMAMARIO ES- TRECHO}

La rigidez de la envoltura de la piel alrededor de la mama es quizás la característica más difícil de corregir de la mama tuberosa hipoplásica. Esta envoltura restringida de la piel no solo interfiere con la colocación de un implante mamario de tamaño adecuado, sino que la falta de desarrollo de un montículo mamario normal no logra estirar la piel adecuadamente, lo que da como resultado un pliegue inframamario anormalmente alto y estrecho. En esencia, se debe crear quirúrgicamente un nuevo surco inframamario y se debe superar la deformidad o pliegue asociado con el pliegue anterior para crear un contorno liso del polo inferior. El manejo exitoso de este aspecto de la anomalía mamaria tuberosa determinará, en gran parte, la calidad del resultado estético. El reconocimiento de esta deformidad preoperatoriamente puede ayudar a preparar al cirujano para lo que vendrá en el momento de la cirugía y permitir que se realice una planificación técnica preoperatoria adecuada. Además, se le puede avisar al paciente con anticipación sobre la naturaleza de su deformidad, lo que luego permite establecer expectativas razonables ${ }^{16}$.

Se pueden emplear varias técnicas para expandir el polo inferior constreñido y para disminuir el surco inframamario alto y estrecho ${ }^{17}$ :

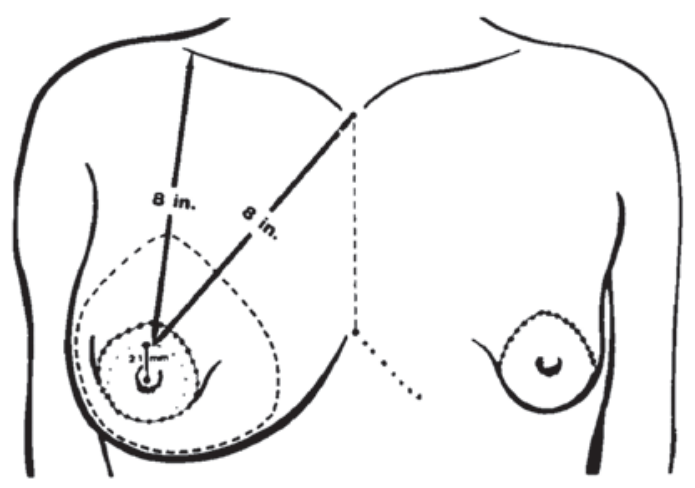

Figura 6.

La parte inferior de la mama debe ser liberada con incisiones radiadas del parénquima. De esta forma, los anillos fibrosos horizontales se cortan y el polo inferior se vuelve más dócil. La profundidad de las incisiones en el parénquima se evalúa visualmente y por palpación. A veces, estas bandas son muy gruesas y están bien representadas, y es posible que sea necesario realizar una incisión en el parénquima muy profundo, cerca de la dermis, teniendo cuidado de no adelgazar demasiado el área. Las incisiones radiadas se puede realizar desde el abordaje inframamario, con una técnica de plano dual o desde el abordaje periareolar.

Se pueden desarrollar diferentes colgajos del parénquima glandular con el fin de dar forma al polo inferior de la mama. Al incidir el parénquima, las bandas fibrosas se interrumpen y el polo inferior se relaja.

Los implantes anatómicos expanden más el polo inferior que los redondos. Es por eso que se prefieren los implantes anatómicos de alto perfil en las mamas tuberosas, aunque algunos cirujanos consideran que se pueden utilizar cualquiera de los implantes, de perfil alto o medio.

La colocación subglandular del implante aplica fuerza directa sobre el polo inferior y permite que se produzca más relajación.

El enfoque periareolar permite un enfoque más fácil y directo para restringir la liberación de bandas y también para reducir el surco inframamario. El surco inframamario está mejor controlado desde arriba que desde abajo, como en el enfoque inframamario ${ }^{16}$.El abordaje periareolar también se prefiere en los senos tuberosos porque también permite tratar el diámetro aumentado de la areola y la herniación del parénquima a través de él ${ }^{17}$.

\section{HERNIACIÓN AREOLAR}

La areola agrandada se baja al diámetro normal de 38 $42 \mathrm{~mm}$ mediante una extracción en forma de rosquilla y la técnicade Round Block. El método permite extender el parénquima herniado lejos de la región areolar. Además, las areolas típicamente lateralizadas se 


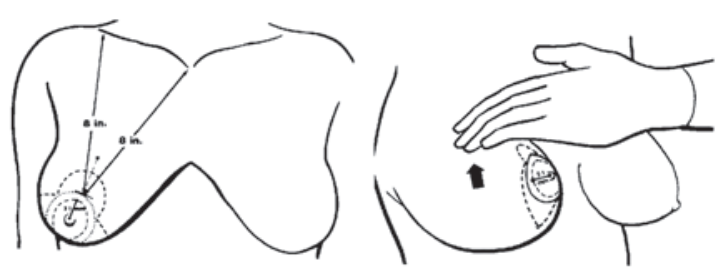

Figura 7.

pueden medializar a través de la desepitelización periareolar. La sutura utilizada en el Round Block puede ser Gore-Tex o nylon 2-0 o 3-0 y se puede utilizar la técnica de entrelazado descrita por Hammond ${ }^{16}$.

\section{ASIMETRÍA MAMARIA}

La asimetría es muy común en los senos tuberosos. Las formas leves de asimetría pueden abordarse utilizando diferentes volúmenes y / o formas de los implantes mamarios en caso de hipoplasia mamaria. Con tipos más severos de asimetrías, hay una mama hipoplásica y una agrandada, ptótica que generalmente muestra las características de una mama tuberosa (surco inframamario alto y estrecho con areola agrandada, desplazamiento medial o lateral). La actitud en tales casos es la primera colocación de un implante en la mama hipoplásica y la reducción con el levantamiento de la mama ptótica agrandada. Si la mama ptótica solo necesita mastopexia, se puede usar el método de auto-aumento, con la creación de un pedículo inferior anclado en la pared torácica. Esto evita la recurrencia de la ptosis, llena el polo superior e interrumpe los anillos fibrosos constrictivos. Si la mama ptótica necesita reducción, se puede elegir una técnica de pedículo superior o pedículo supero-medial con una cicatriz vertical o $\mathrm{T}$ invertida dependiendo del grado de ptosis ${ }^{16}$. La simetría es muy difícil de lograr justo después de la primera cirugía y el paciente debe ser advertido de antemano.

\section{DISCUSIÓN}

No hay una sola técnica quirúrgica que pueda ser aplicada en todos los casos de mama tuberosa. Más bien, el cirujano debe poseer un arsenal de técnicas de las que pueda elegir cuando se enfrente con varios tipos de mamas tuberosas. Diferentes autores propusieron a lo largo del tiempo una serie de métodos para tratar este tipo de patología.

Una de las primeras técnicas quirúrgicas utilizadas fue propuesta por Williams $(1972)^{18}$ quien propone la realización de un aumento de volumen con implantes ma-
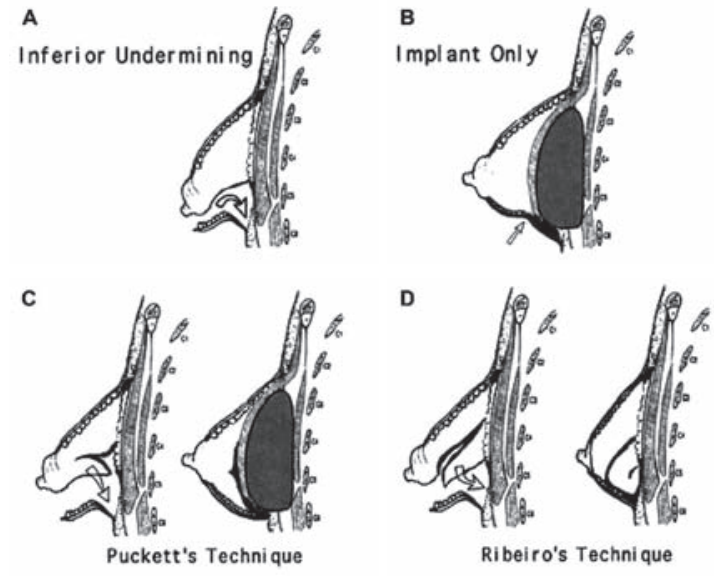

Figura 8. Técnicas para liberar la base contraída; (A) disección subcutánea y puntuación, (B) disección de bolsillo de implante, (C) colgajo basado en areola, (D) colgajo de pared de tórax (3).

marios de silicona a nivel prepectoral. Estos implantes eran redondos de superficie lisa. No se realizaba modificación del anillo constrictor mamario y al utilizar implantes redondos se producía un doble surco (double boubble). Los resultados de este procedimiento no reportaron satisfacción en las pacientes por no corregir las deformidades mamarias presentes (Figura 5).

S. Aston y T. Rees ${ }^{1}$ proponen un abordaje a nivel del surco submamario y la posterior realización de incisiones radiales en toda la base mamaria, con la colocación de prótesis de silicona redondas a nivel retromamario y si es necesario la resección de piel periareolar para la corrección de la protrusión de tejido a nivel areolar. Esta técnica genera cicatrices notorias o distróficas a nivel inframamario, además de no corregir el doble surco inframamario, tampoco proporciona una longitud adecuada entre la areola y el nuevo surco inframamario.

Toranto ${ }^{19}$ propone una cirugía en dos tiempos para la corrección de mamas tuberosas tipo I y II, durante el primer tiempo realiza un aumento trans-mamario con una prótesis redonda con la finalidad de crear volumen mamario y así aumentar los diámetros mamarios. Cuatro semanas más tarde si no hay complicaciones, se realiza un segundo tiempo que consiste en la corrección de la ptosis mamaria de la mama previamente expandida. En este segundo tiempo se realiza un marcaje similar al propuesto por Arie-Pitanguy ${ }^{20}$ más una reducción de tejido mamario protuberante presente. Esta técnica deja como secuela grandes cicatrices en las mamas, además de tener que realizar en dos tiempos quirúrgicos bajo anestesia general. Esta técnica puede dejar pinzamiento en los polos inferiores por falta de tejido, con un resultado poco estético de las mamas (Figuras 6 y 7$)$..

Puckett ${ }^{21}$ propone una incisión periareolar que permite la remodelación de la areola con un acceso a la glándula y remodelación de la misma con un colgajo en libro abierto, más la colocación de prótesis redonda por debajo de este colgajo. La liberación de la zona subma- 


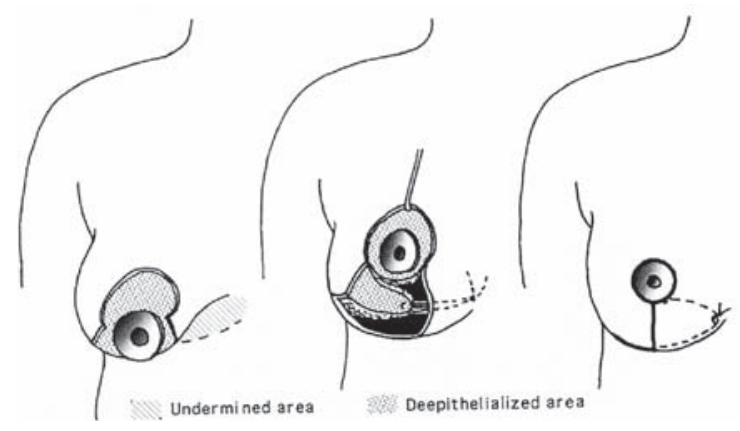

Figura 9.

maria permite reposicionar la mama y conseguir el descenso del surco sub-mamario a un nivel ideal o normal (sexta costilla).

La técnica propuesta por Liacyr Ribeiro ${ }^{22}$ para la corrección de mamas tuberosas grado III donde mediando una incisión periareolar, seguido de una incisión y división del tejido mamario en dos porciones(tejido glandular por abajo del área subareolar y tejido glandular por encima del área subareolar) logra una ruptura del anillo constrictor y un colgajo de pedículo inferior para dar volumen, sin utilizar prótesis (Figura 8). Grolleau (1999) propone un procedimiento para tratar mamas tuberosas grado I. utilizando una mastoplastia con un pedículo superior y un colgajo dermoglandular ínfero-lateral para llenar el cuadrante ínfer-medial que es el área de mayor deficiencia de tejido glandular en las mamas tuberosas grado I. presentando frecuentemente necesidad de revisión secundaria de los $\operatorname{casos}^{3}$ (Figura 9).

Tebbets $^{21}$ (2001) Con el tipo III de plano dual (polos inferiores constrictos), la cobertura muscular superior se conserva pero el borde inferior del musculo se mueve superiormente, removiendo cualquier restricción muscular del polo inferior a la expansión. El cirujano tiene un acceso ideal a la superficie posterior del parénquima para incidir y redistribuirla, y el implante puede optimizar la redistribución del parénquima, expandir la envoltura y corregir el polo inferior constrictivo. Mandrekas ${ }^{2}$ diseca la piel de la mitad inferior de la mama hasta la fascia del pectoral con un abordaje periareolar. Una vez que se alcanza el surco inframamario, la disección continúa detrás del seno y la glándula se exterioriza a través de la abertura periareolar. Se secciona transversalmente la mitad inferior del seno con una incisión vertical, dividiendo el anillo fibroso constrictivo. Los pilares resultantes se aproximan de forma aproximada o se les permite redistribuir libremente. Si es necesario, se coloca un implante mamario de silicona detrás del parénquima mamario, con cuidado especial para que los pilares cubran el implante en su totalidad (Figura 10).
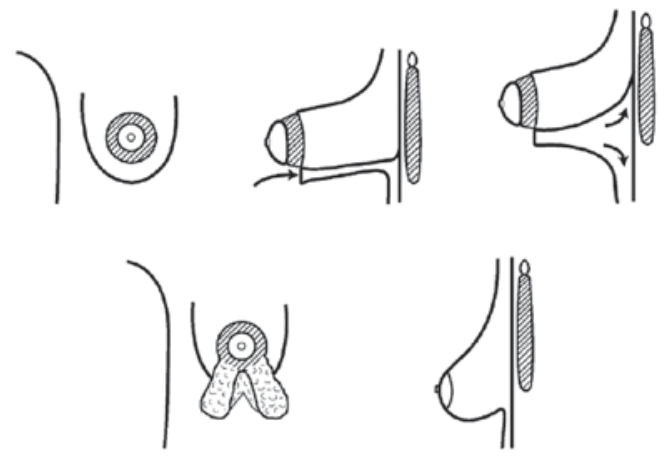

Figura 10.

Persichetti ${ }^{22}$ utiliza colgajos parenquimatosos medial o lateralmente para rellenar los polos inferiores deficientemente lateral o medialmente inferiores de la mama tuberosa .

Foustanos utiliza un enfoque en dos etapas con una combinación de expansor tisular e implantes de silico$\mathrm{na}^{23}$. En la creencia del autor, el expansor de tejido anatómico agranda la base mamaria sin ninguna cicatriz adicional y evita la deformidad doble pliegue.

Botti usa una mastopexia periareolar y un aumento de senos con implantes con expansión mamaria para superar el polo inferior constreñido. Los implantes se alojan en el lugar submuscular después de un desprendimiento completo de las inserciones inferiores del músculo pectoral y la porción inferior de la glándula se separa de la piel y del músculo. La glándula expandida que usa varias incisiones cubre el implante y está anclada al surco inframamario con puntos transcutáneos ${ }^{24}$. Muti crea diferentes colgajos glandulares que se movilizan desde el área relativa excedente a las partes más deficientes de la mama. Los colgajos glandulares, cuadrangulares o triangulares, se disecan con incisiones perpendiculares y se rotan profundamente con el ápice hacia el nuevo surco inframamario y se fijan con suturas no absorbibles. La mama hipoplásica aumenta con una prótesis de silicona. Las cicatrices resultantes son periareolares, intraareolares o asociadas a una vertical 25.

Serra-Renom utiliza una técnica modificada de Puckett. La glándula se exterioriza después de una disección subcutánea y retroglandular, y se despliega creando un colgajo de base inferior de la glándula que parece un libro abierto. La técnica se utiliza para senos tuberosos severos de grado III y se combina con la colocación de un implante cubierto por la glándula desplegada. Después de 6 meses, si la doble burbuja creada por el anillo de constricción no desaparece, el injerto de grasa se realiza con buenos resultados ${ }^{26}$.

A pesar de las presentaciones clínicas variables que pueden tener las mamas tuberosas, existen algunas deformidades comunes que deben corregirse en todos los casos. La falta 
de corrección de estas deformidades conduce a resultados insatisfactorios y una tendencia a la recurrencia. Estos $\operatorname{son}^{17}$ :

- Movilización del parénquima mal posicionado de áreas de exceso de tejido a áreas de deficiencias tisulares utilizando diferentes colgajos glandulares con el fin de remodelar la mama.

- Liberación de la base mamaria estrechada (expansión de la base mamaria descrita por Botti).

- Liberación del polo inferior estrecho.

- Tratamiento de la deficiencia del volumen.

- Manejo del surco inframamario alto y estrecho.

- Alteraciones de la areola.

Varios problemas son importantes y deben tenerse en cuenta:

1. La deformidad debe corregirse antes de la colocación del implante. Si el implante se coloca sin corregir la deformidad, se acentuará la apariencia snoopy de las mamas.

2. Los implantes grandes deben evitarse en las mamas tuberosas.
3. Los implantes anatómicos con proyección alta son una buena opción porque ayudan en la relajación del polo inferior. Hay autores que igualmente recomiendan implantes redondos, ya sea de alto perfil o de perfil moderado ${ }^{25}$.

\section{CONCLUSIÓN}

La mama tuberosa presenta un gran reto para los cirujanos plásticos, a lo largo del tiempo se han descritos numerosas técnicas quirúrgicas pero no hay una sola técnica quirúrgica que pueda ser aplicada en todos los casos de mama tuberosa. El reconocimiento de esta deformidad preoperatoriamente puede ayudar a preparar al cirujano para lo que vendrá en el momento de la cirugía y permitir que se realice una planificación técnica preoperatoria adecuada. El uso de un sistema de clasificación permite una racionalización de la indicación de las diferentes técnicas quirúrgicas.

\section{BIBLIOGRAFÍA}

1. Rees TD, Aston SJ. The tuberous breast. Clin Plast Surg 1976; 3: 39347.

2.. Mandrekas AD, Zambacos GJ, Anastasopoulos A, Hapsas D, Lambrinaki N, loannidou-Mouzaka L. Aesthetic reconstruction of the tuberous breast deformity. Plast Reconstr Surg. 2003;112 (4):1099-108.

3. Grolleau JL, Lanfrey E, Lavigne B, Chavoin JP, Costagliola M.:Breast base anomalies: Treatment strategy for tuberous breasts, minor deformities, and asymmetry. Plast. Reconstr. Surg 1999, 104(7): 2040.

4. Muti E. The tuberous breast. Florence: SEE-Firenze; 2010.

5. Oroz J, Pelay MJ, Escudero FJ. Reconstrucción de la mama tuberosa. An. Sist. Sanit. Navar. 2005; 28 (Supl. 2): 101-108.

6. Teimourian B, Adhman MN. Surgical correction of the tuberous breast. Ann Plast Surg 1983; 10: 190-193.

7. Kolker AR, Collins MS, . Tuberous Breast Deformity: classification and Treatment Strategy for Improving consistency. Aesthetic Correction. Plast Reconstr Surg 135(1):73-86, 2015.

8. Neligan P.C. Volumen cinco-Tomo II-Tercera edición. Cirugía Plástica: Mama. 23.1. Anomalías congénitas de la mama. Egle Muti. Pag 521. 2013.

9. Nahai $F$. The art of aesthetic surgery principles and techniques. St Louis: Quality Medical; 2005:2486-2488.

10. Hughes LE, Mansel RE, Webster DJT. Benign Disorders and Diseases of the Breast: Concepts and Clinical Management. London: Bailliere Tindal, 1989. Pp. 5-14.

11. Osborne MP. Breast development and anatomy. Breast Diseases, 2nd Ed. Philadelphia: J. Lippincott Co., 1991. Pp. 1-13

12. Bostwick J III. Anatomy and physiology. Plastic and Reconstructive Breast Surgery, 2nd Ed. St. Louis, Mo.: Quality Medical Publishing, 2000. Pp. 89-93.

13. Moore KL. The integumentary system. The Developing Human: Clinically Oriented Embryology, 4th Ed. Philadelphia: Saunders, 1988. Pp. 426-428.

14. Von Heimburg D, Exner K, Kruft S, Lemperle G. The tuberous breast deformity: classifi cation and treatment. Br J Plast Surg. 1996;49 (6):339-45
15. Brown MH, Somogyi RB. Surgical Strategies in the Correction of the Tuberous Breast. Clin Plast Surg. 2015.

16. Hammond D. Management of the tuberous breast. Atlas of aesthetic breast surgery. Philadelphia: Saunders Elsevier; 2009.

17. Isac A, Isac C. Tuberous Breast. Aesthetic Surgery of the Breast.2015

18. Williams JC. Experience with a large series of Silastic Breast implants. Plast Reconst Surg . 49:253. 1972.

19. Toranto IR. Two-Stage Correction of Tuberous Breast. Annual Meeting of the American Plastic Surgery. 1980.

20. Ely JF. Reduction mammaplasty- An eclectic approach. Aesthetic Plast Surg. 1978:2 (1):95-101.

21. Puckett CL, Concannon MJ. Augmenting the narrow based breast: the unfurling technique to prevent double-bubble deformity. Aesthetic Plast Surg. 1990;14 (1):15-9.

22. Riberiro L. Canzi W, Buss A. Tuberous breast: a new approach. Plast Reconst Surg. 1998;110 (1):42-50.

21. Tebbetts JB. Dual plane breast augmentation: Optimizing implant-soft-tissue relationships in a wide range of breast types. Plast Reconstr Surg. 2001;107:1255-1272.

22. Persichetti P, Cagli B, Tenna S, Simone P, Marangi GF, Li Vecchi G. Decision making in the treatment of tuberous and tubular breasts: volume adjustment as a crucial stage in the surgical strategy. Aesthetic Plast Surg. 2005; 29:482-8.

23. Foustanos A, Zavrides H. Surgical reconstruction of tuberous breasts. Aesthetic Plast Surg. 2006; 30(3): 294-300.

24. Botti G. Periareolar mastopexy with glandular expansión and implants. In: Botti G, editor. Aesthetic mammoplasties: practical atlas of plastic surgery. Florence: SEE-Firenze; 2008. p. 361-81.

25. Muti E. Local flaps for tuberous and asymmetric breasts. In: Hall-Findlay EJ, Evans GRD, editors. Aesthetic and reconstructive surgery of the breast. Philadelphia: Saunders Elsevier; 2010.

26. Serra-Renom JM, Muñoz-Olmo J, Serra-Mestre JM. Treatment of grade 3 tuberous breasts with Puckett's technique (modified) and fat grafting to correct the constricting ring. Aesthetic Plast Surg. 2011; 35(5): $773-81$. 